INTERNATIONAL DESIGN CONFERENCE - DESIGN 2018

https://doi.org/10.21278/idc.2018.0447

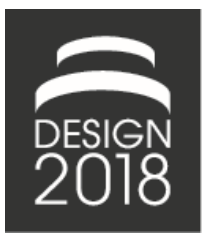

\title{
TRI-WHEEL STAIR WALKER: DESIGN PROPOSAL OF AUXILIARY WALKER USABLE AT DISTRICT INCLUDING STAIRWAY
}

\author{
M. Le and E.-C. Jung
}

\begin{abstract}
This study is to suggest a new auxiliary walker which 1) can be used not only on flat but also in real urban environments; 2) can be used without psychological burden when going outside; and 3) can be manufactured with low cost to improve the mobility of the elderly and to lead to a healthy and pleasurable life. We investigated the characteristics of the movement of the elderly and proposed the design concept Tri-Wheel Stair Walker based on interviews with and observation of elderly users. The concept is expected to be a safe and cheap solution to the door-to-door mobility in a smart city.
\end{abstract}

Keywords: human centred design, industrial design, product structure, mobility, auxiliary walker

\section{Introduction}

Today, a widely shared objective is the provision of seamless door-to-door multimodal mobility. Despite many technological improvements, gaps still exist in the transport chain (Bonneville, 2016). The provision of door-to-door transport tends to be of greater importance to older disabled people than to others, because even though the transport is accessible to ordinary people, frailties associated with ageing may make it difficult to address the gaps (AGE Platform Europe, 2002). With the growing awareness of Right to Access, it has become common to build a slope instead of stairs to secure a passageway, but stairs still exist, and it is hard to say that the slope is safe for those who have difficulty walking.

When a person is aged or is temporarily weakened due to an accident or illness, he or she will have difficulty walking. Furthermore, many people experience difficulty walking up and down stairs, even though they can walk on level ground without any effort. The main differences between walking up and down stairs and walking on level ground are that each step initiates on the toes and ball of the foot rather than the heel, and the vertical transfer of weight requires either extra effort in ascent or a controlled fall in descent (Roy, 2001).

People use auxiliary walkers when their muscles become weak. Many different products can be used to assist with walking, such as canes, walkers and wheelchairs. However, canes are only available to people who are relatively strong, and walkers currently available are not able to fully consider short flights of stairs or slopes, so mobility is still limited in environments with stairs. Additionally, due to the expense associated with walkers, many elderly people place heavy things in used baby carriages and use them as auxiliary walkers. This leads to bad positioning and causes physical pain.

In this context, the purpose of this study is to suggest a new auxiliary walker which 1) can be used not only on flat but also in real urban environments; 2) can be used without psychological burden when 
going outside; and 3) can be manufactured with low cost to improve the mobility of the elderly and to lead to a healthy and pleasurable life.

\section{Process and methods}

To achieve its purpose, this study was conducted in five stages.

First, we reviewed related studies to understand the characteristics of the elderly walking up and down the stairs, to categorize the assistive products, and to analyse the advantages and the problems associated with these products.

Second, we interviewed users who have walking problems and who are in urgent need of door-to-door mobility, and we observed how they use their auxiliary walkers to address real needs.

Third, we suggested a concept that can be used when walking on level ground, walking up the stairs, and walking down the stairs, created a prototype, and implemented a pilot test.

Fourth, we created two other prototypes to evaluate the entire structure and details of the concept, and we further developed the design.

Finally, we completed the design and proposed the Tri-Wheel Stair Walker design concept using computer simulation.

\section{Preliminary studies}

\subsection{Characteristics of walking and vertical movement of the elderly}

Mobility activities include various activities related to movement in one's daily life, from awakening to starting activities to going back to bed. According to a study by Jung et al. (2007), it can be said that lying down, moving in bed, sitting down and standing up (from a bed, chair or wheelchair), moving between a bed and a toilet, going up and down stairs, going out by walking or with a wheelchair, using the escalator, and taking the bus (train) are all included in mobility activities. Therefore, it is necessary to classify them and analyse their characteristics, and to develop suitable methods to assist in these activities according to the results.

According to the Modified Barthel Index (MBI) and the Functional Independence Measure (FIM), the Barthel ADL indices, which are widely used to measure the functional independence in activities of daily living, human mobility activities in daily life can be divided into three categories: horizontal movement by walking or using a wheelchair; vertical movement, such as going up and down stairs; and movement between beds, chairs and toilets, which involves sitting down and standing up (Fricke and Unsworth, 1997). The characteristics of each activity can be seen below (Table 1).

Table 1. Classification of mobility activities and their characteristics

\begin{tabular}{|l|l|}
\hline Mobility Activities & Characteristics \\
\hline Locomotion & Transferring from one place to another by walking or other methods \\
\hline Stair Climbing & Activities that causes a change in vertical position \\
\hline Chair/Bed Transfer & Movement involving sitting down and standing up \\
\hline
\end{tabular}

According to a survey (Lee, 2008) of elderly people aged 60 years and older living in Seoul, South Korea, most of the respondents have difficulties in climbing stairs and require assistance. However, most of the survey participants indicated that they do not experience much difficulty in walking on flat ground or sitting down and standing up from chairs.

Vertical movement means that the flexion and extension of the lower limbs must be repeated and such upward and downward movements are accompanied by a significant level of muscle activities. Although the degree of muscle activity may depend on walking speed, research shows that it takes about 10 to 15 times more energy to walk up and down stairs than to walk on the level. Elderly people tend to keep their centre of body weight more backward to stabilize their posture because they are less able to maintain their balance when going up and down stairs. Gravity accelerates their walking speed when going down stairs and this leads to fluctuating balance, which causes anxiety about falling (Kim et al., 2012). 
Considering the characteristics of stair walking and the difficulty of elderly people's mobility, supporting stair walking through walking aids should be a primary issue under discussion.

\subsection{Walking aid classification and analysis}

A walking aid is designed for those who have difficulty in maintaining balance after standing due to walking disorders, helping individuals to maintain walking safety by supporting their weight while walking. In other words, it is designed to assist in safely walking, both indoors and outdoors, for the elderly and the handicapped, who have difficulty walking due to weaknesses in strength and muscles. By using a walking aid, the elderly can not only enhance their confidence in walking and eliminate the fear of falling, but also gain confidence in daily life because they can live more independently with the help of auxiliary walkers. Walking aids should provide both psychological benefits, such as confidence and independence in daily life, and biomechanical advantages.

Walking aids such as canes, walkers, and wheelchairs are employed depending upon the degree of walking difficulty of elderly people. A cane, which is most widely used, may cause imbalanced posture and require users to have the capability of maintaining balance. Elderly people who have more difficulty walking may use a walker, but many users psychologically reject walkers because they think that walkers make them look like patients. If one can barely walk, he or she will have to use a wheelchair. While many elderly people do not experience great difficulty when walking on level ground, they need assistance when walking up and down the stairs. Therefore, the elderly who can walk alone require an auxiliary walker to help them maintain good posture for balanced walking because of their lowered perception and cognitive capability. However, more importantly, they need an auxiliary walker that they can rely on when walking up stairs that can also help them maintain balance and walk safely when going down stairs.

While canes can be used on stairs, there is a disadvantage, because they do not help to maintain balance. On the other hand, a walker can be a safe aid to walking on flat terrain and on slopes, but when walking on stairs, it becomes a burden. Therefore, in this study, we strive to propose a design for an auxiliary walker that combines the advantages of both canes and walkers, with the target position shown below (Figure 1).

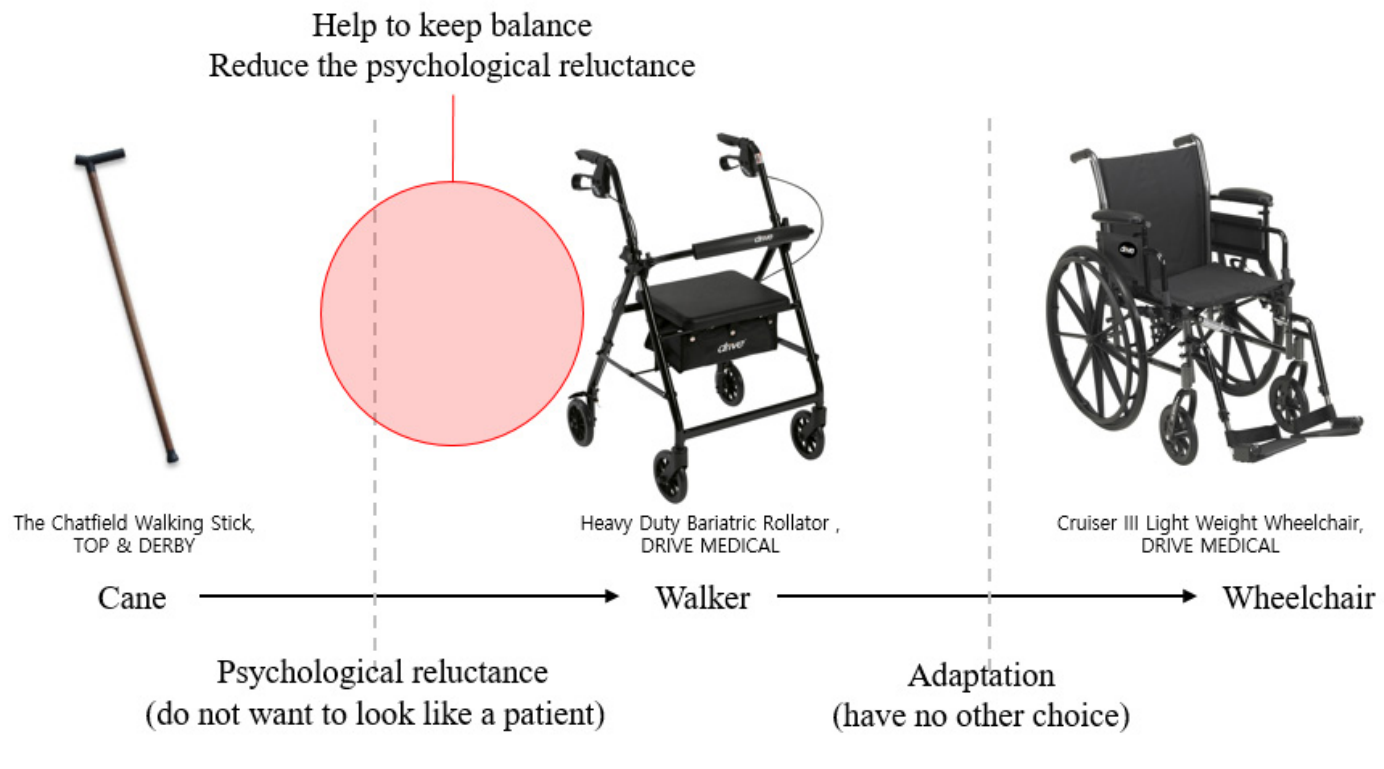

Imbalanced position

Insufficient physical activity

Figure 1. Classification of walking aids and target position 


\section{Needs analysis}

To investigate the actual usage situation and determine the needs of users we interviewed the elderly users in Shanghai, China and Seoul, South Korea. Considering that elderly users may not have enough awareness and concentration to finish the interviews, the interviews were combined with observations. Through these interviews and observations, we believe that we can grasp the problems and difficulties when the elderly use their walking aids and determine their real needs, which are closely related to their daily lives. The interviews were conducted between December 2016 and March 2017 and included five interviewees (Table 2).

Table 2. List of interviewees

\begin{tabular}{|l|l|l|l|l|l|l|}
\hline & Nationality & Gender & Age & Household type & Disease/disorder & Walking aids \\
\hline 1 & China & Female & 63 & Living with spouse & Recovery after stroke & Walker \\
\hline 2 & China & Male & 68 & Living alone & $\begin{array}{l}\text { High blood pressure, } \\
\text { Kyphosis }\end{array}$ & Walker \\
\hline 3 & China & Female & 83 & Living with children & Kyphosis & Cane \\
\hline 4 & Korea & Female & 65 & Living with children & Backache & $/$ \\
\hline 5 & Korea & Male & 78 & Living with spouse & Recovery after stroke & walker \\
\hline
\end{tabular}

Although the interviewees did not want to use the walking aids at first because they thought these products would make them look like patients, most users attained practical benefits through actual use and came to realize that walking aids are useful tools to help them to live independently. So for the users who have never used walking aids before, our approach should not give an image that the product is designed for the patient. It should be well designed to eliminate users' psychological burden, inducing them to use the aid. It should also be easy and safe to use, and it should eliminate users' anxiety by making users believe that it can prevent them from the danger of falling. Eventually, it will make users form a psychological attachment through long-term use.

We also observed how users employ walking aids outdoors during the interview. In particular, since there is not only flat ground, but also stairs and slopes in the urban environment, we intensively observed the problems of using the walking aids in such environments. The results can be seen in Figure 2.
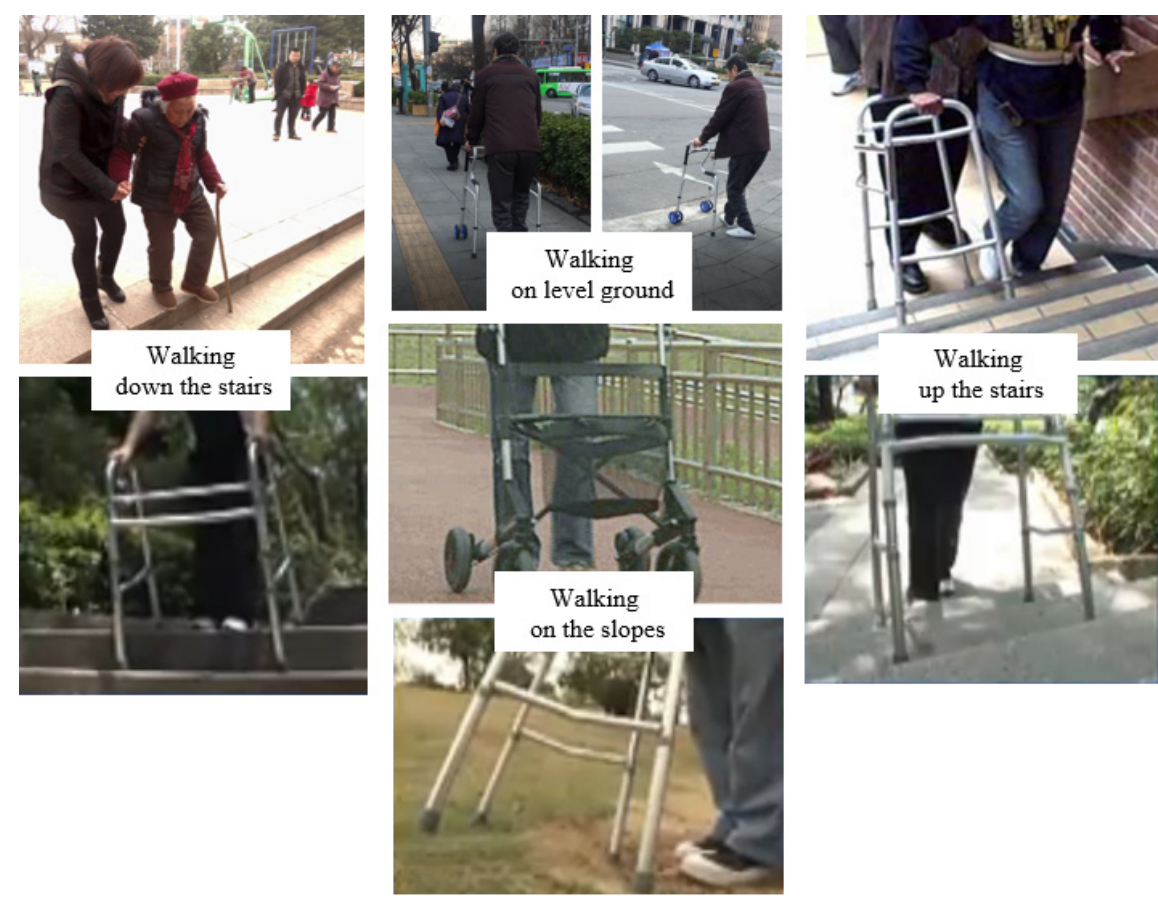

Figure 2. Walking with walking aids 
Through these observations, we found that elderly users were more careful when they went up and down the stairs than when they were walking on flat ground because of the fear of falling. Walkers did not work at all on the stairs, instead becoming a burden or even a risk factor. Walkers with wheels are particularly dangerous because if the wheels roll down the stairs, users may be injured. Canes can help users to walk on stairs to some extent, but they still require other support such as railings. Therefore, this study strove to propose an auxiliary walker that can relieve the fear of falling and provide stable support in urban environment with stairs and slopes. The wheels should be unlocked when used on the stairs.

\section{Design concept and pilot test}

Based upon research related to the characteristics of vertical movement of the elderly, as well as user research, the design concept is proposed $n$ Figure 3.

The structure of the walker with one front wheel and two rear wheels can provide stable support while walking. The height can be adjusted according to the mode change, so that the handle can be held in a comfortable position after switching the mode. The angle of the frame can be changed with the mode change, so that various stair heights can be adjusted. When walking up the stairs, the rear wheels are fixed so that the user will not fall backward. When walking down the stairs, the front wheel is fixed so that the user will not fall forward and can use the walker safely.

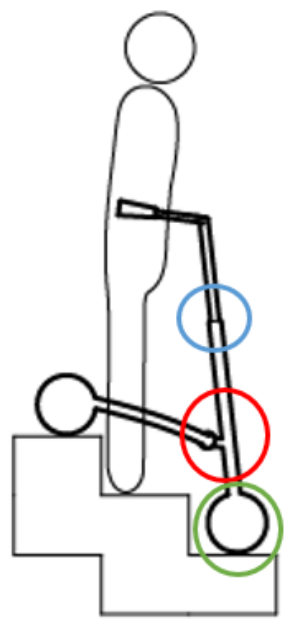

Walking

down the stairs

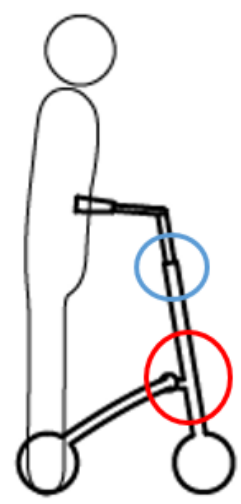

Walking

on level ground

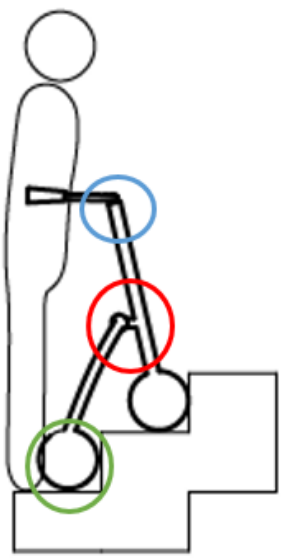

Walking

up the stairs

Figure 3. Basic switching frame of stair climbing mode and walking mode

To test the feasibility and practicality of the concept, we created a prototype and conducted a pilot test. The frame of the prototype was printed by $3 \mathrm{D}$ printer and lockable wheels were installed so that we could switch the basic mode. The process of the pilot test and the status values required three modes (walking up the stairs, walking down the stairs, and walking on level ground), which can be seen in Figure 4.

Through the pilot test, we found that 1) the concept we proposed can help to maintain balance better than canes on both sides; 2) it has the effect of reducing the burden on the knees because it can be relied upon when going upstairs; and 3) it can be used safely when going downstairs because the front wheel is fixed so that it will not roll down the stairs. However: 1) the walker is heavier than a cane demanding relative strength in both hands; and 2) it is important to consider a natural interface to switch the modes. In addition, we gained the additional insight that it should be easy to store in indoor spaces or in automobiles so that door-to-door mobility can be realized during use.

Based on this pilot test, our design development direction is as follows: 1) design the control unit that can be switched to the three modes of walking up the stairs, walking down the stairs, and walking on level ground; and 2) develop the collapsible structure and make the walker easy to store at home or in automobiles. Also included in the details, including the moderately light material, the ergonomic structure of the handles should be carefully considered. 


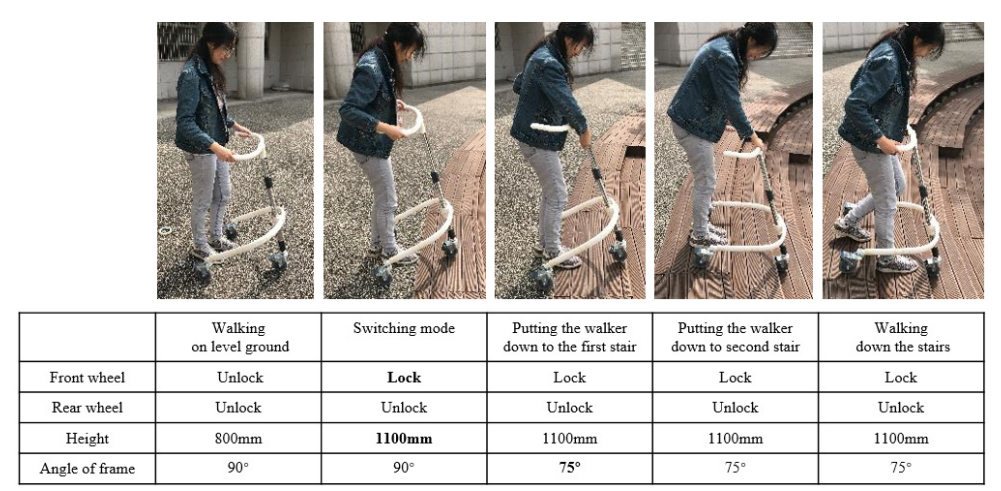

Walking on level ground $\rightarrow$ Walking down the stairs
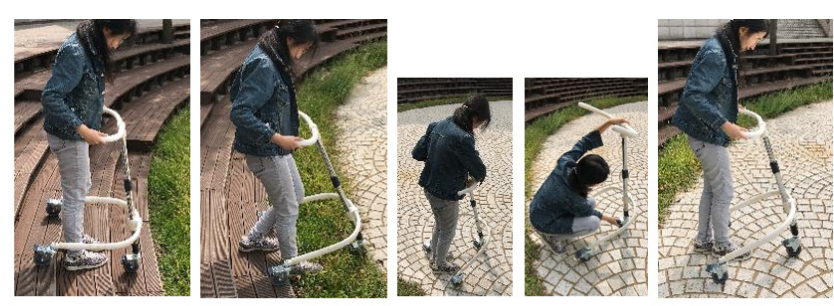

\begin{tabular}{|c|c|c|c|c|}
\hline & $\begin{array}{c}\text { Walking } \\
\text { down the stairs }\end{array}$ & Walking down the last stair & Switching mode & $\begin{array}{c}\text { Walking } \\
\text { on level ground }\end{array}$ \\
\hline Front wheel & Lock & Lock & Unlock & Unlock \\
\hline Rear wheel & Unlock & Unlock & Unlock & Unlock \\
\hline Height & $1100 \mathrm{~mm}$ & $1100 \mathrm{~mm}$ & $\mathbf{8 0 0 \mathrm { mm }}$ & $800 \mathrm{~mm}$ \\
\hline Angle of frame & $75^{\circ}$ & $75^{\circ}$ & $\mathbf{9 0 ^ { \circ }}$ & $90^{\circ}$ \\
\hline
\end{tabular}

Walking down the stairs $\rightarrow$ Walking on level ground

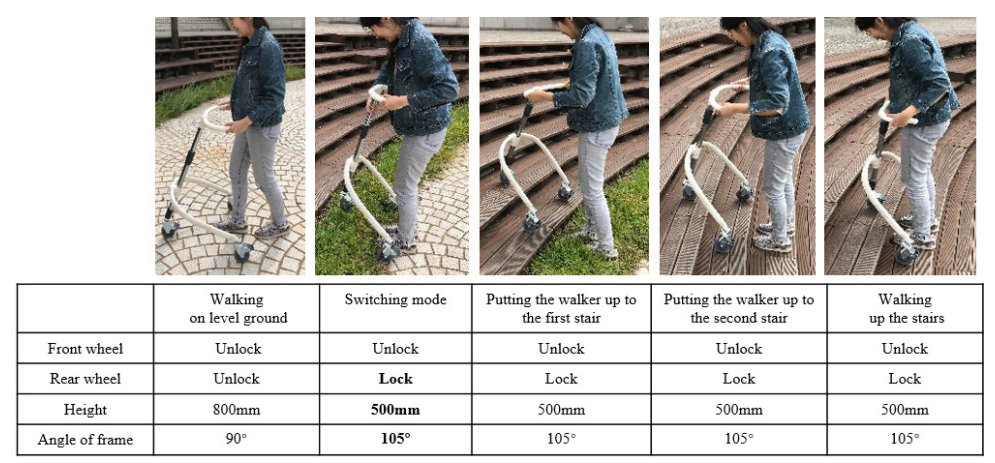

Walking on level ground $\rightarrow$ Walking up the stairs

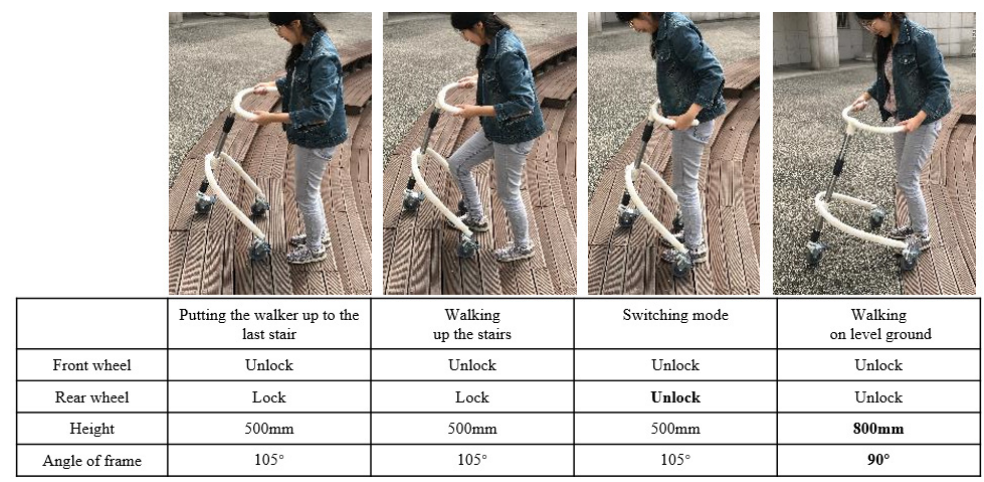

Walking on level ground $\rightarrow$ Walking up the stairs

Figure 4. The process of the pilot test and the status values required for the three modes 


\section{Design development}

A study (Kim et al., 2017) pertaining to the reasons for the non-use of assistive devices demonstrates that the usability of assistive devices is an important determinant in the purchase and utilization of such equipment. The user interface is the means by which a user interacts with the product to enhance usability. The interface of the walker is much simpler than that of electronic devices, but the characteristics of the target user should more carefully be considered.

In this study, the product we proposed is a walker controlled with both hands. It requires the operations of propelling and steering, braking, parking, and adjusting. The handle, which is a propelling device, and the brake, which is a braking device, are close to each other, so that the device can be conveniently used by the elderly. In order to make the interface possible, it is necessary to study the structures of the brake, the wheel, and the frame.

The prototype shown in Figure 4, which was created to conduct the pilot test, has verified the possibility of using the walker safely with the three modes of walking up the stairs, walking down the stairs, and walking on level ground and has also demonstrated what kind of operation is needed. However, the user interface and the structure to realize the interface had not been adequately considered. Therefore, we made two additional prototypes with paper to determine the method of operation and the structure.

\subsection{First paper prototype}

The first paper prototype as shown in Figure 5, was made to intuitively demonstrate the basic form and proportions of the product, and also to show how to switch the modes and fold the product.
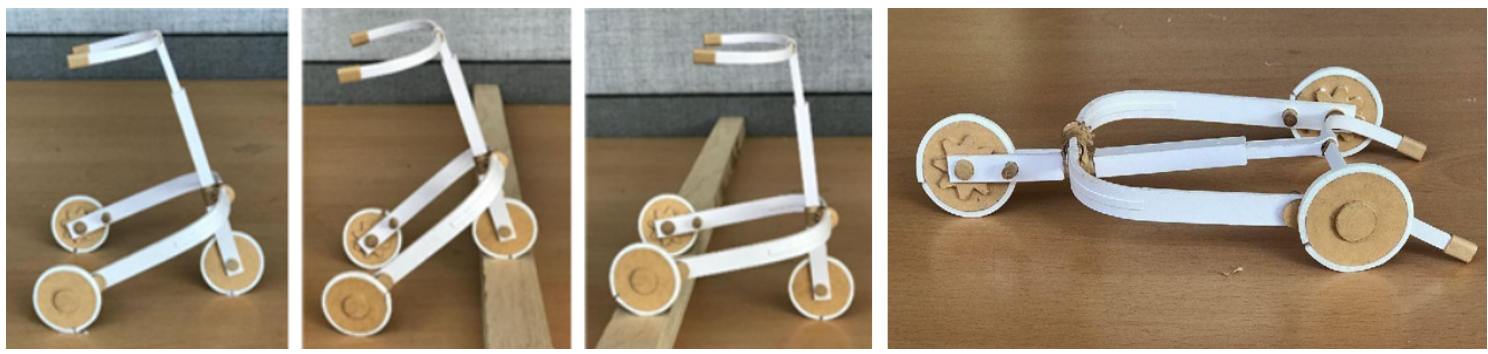

Figure 5. First paper prototype (walking on level ground / walking up the stairs / walking down the stairs / storage mode)

The rotating shaft and fixed structure were made to adjust the angle of the frame (Figure 6, left). This structure allows the frame to be fixed at $75^{\circ}$ (walking up the stairs), $90^{\circ}$ (walking on level ground), $105^{\circ}$ (walking down the stairs), and $180^{\circ}$ (storage). The wheels can be controlled by the installed cog wheels (Figure 6, right).
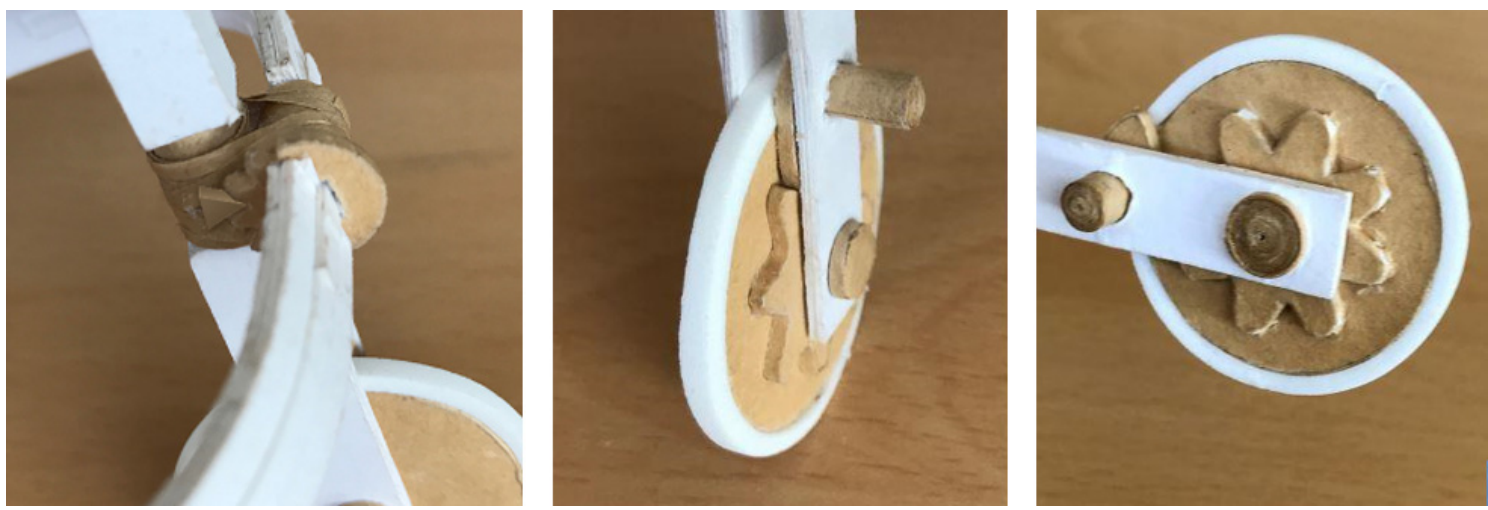

Figure 6. Rotating shaft structure (left) / wheel structure (middle, right)

Through the test using the paper prototype, we identified the following problems.

- The wheels are too thin to be stable. 
- The gear teeth are insufficient, which means that the walker can only be controlled at specific angles. It will be very dangerous if the user cannot stop the walker immediately on narrow stairs.

- The fixing portion on the rotary shaft is too small to support the frame.

- The problem of how to connect the mode switch and the brake has not been solved.

\subsection{Second paper prototype}

In order to solve problems that could not be solved by the first prototype, we made a second prototype, as shown in Figure 7. This prototype was primarily designed to fully understand the structure of the rotary shaft, the brakes and the wheels. Therefore, unlike the prototype shown in Figure 4, Figure 5 and Figure 6, the form and proportions were not considered.
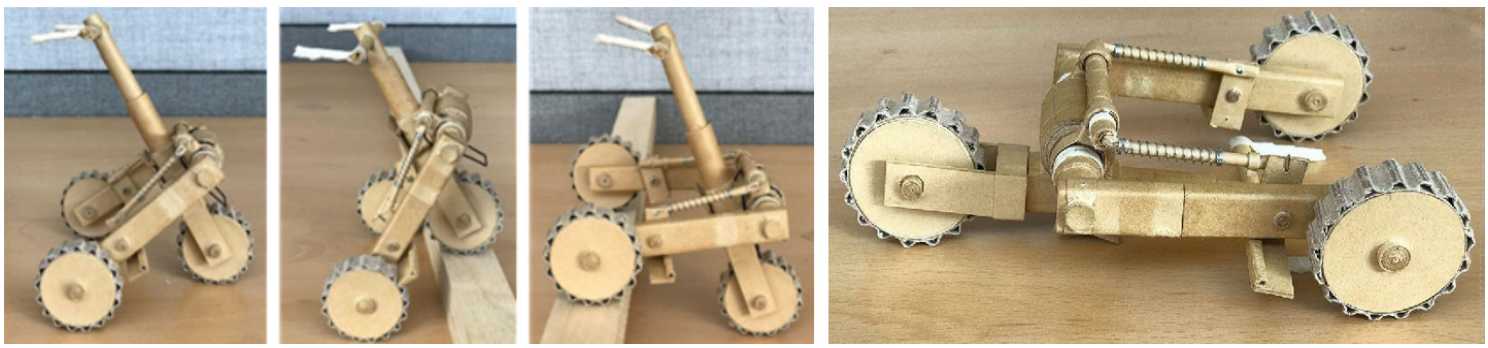

Figure 7. Second paper prototype (walking on level ground / walking up the stairs / walking down the stairs / storage mode)

As shown in Figure 8, for the convenience of braking operations, the wheel cannot be rolled when the brake is pulled forward, and the brake can be released automatically because of the spring. The portion that supports the rotary shaft is designed to be larger, so that the frame can be securely fixed. In addition, the brakes can be locked automatically while the frame angle change. Therefore, safe walking can be ensured.
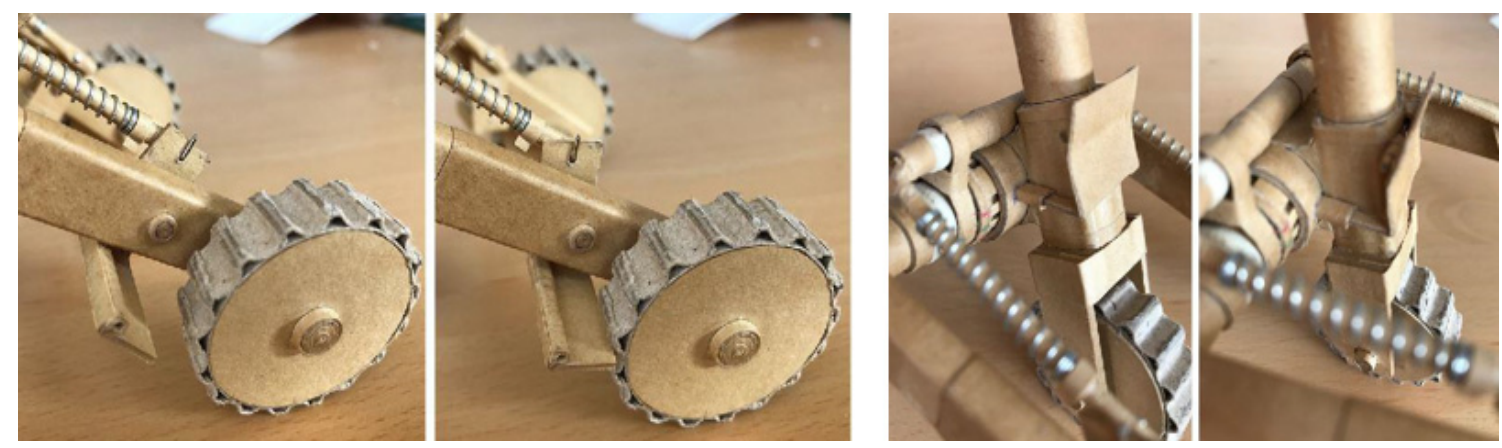

Figure 8. Wheel and brake structure (left) / rotating shaft structure (right)

The second prototype shown in Figure 7 and Figure 8, solved the interface and structural problems of the walker. Most importantly, regarding safe mobility; 1) there is no risk of falling back, even if the rear wheels are not braked, because the rear wheels can go forward only when going up the stairs; and 2) when going down the stairs, not only the front wheel, but also the rear wheels, should be locked together. Therefore, we developed the design that brakes can be locked automatically when switching from the walking on level ground mode to the walking down the stairs mode (see Figure 4), with the angle of the frame changing from $90^{\circ}$ to $75^{\circ}$.

\section{Final design}

Based on the pilot test and two detail prototypes, we proposed the Tri-Wheel Stair Walker (Figure 9) which is expected to be a solution to door-to-door mobility. 


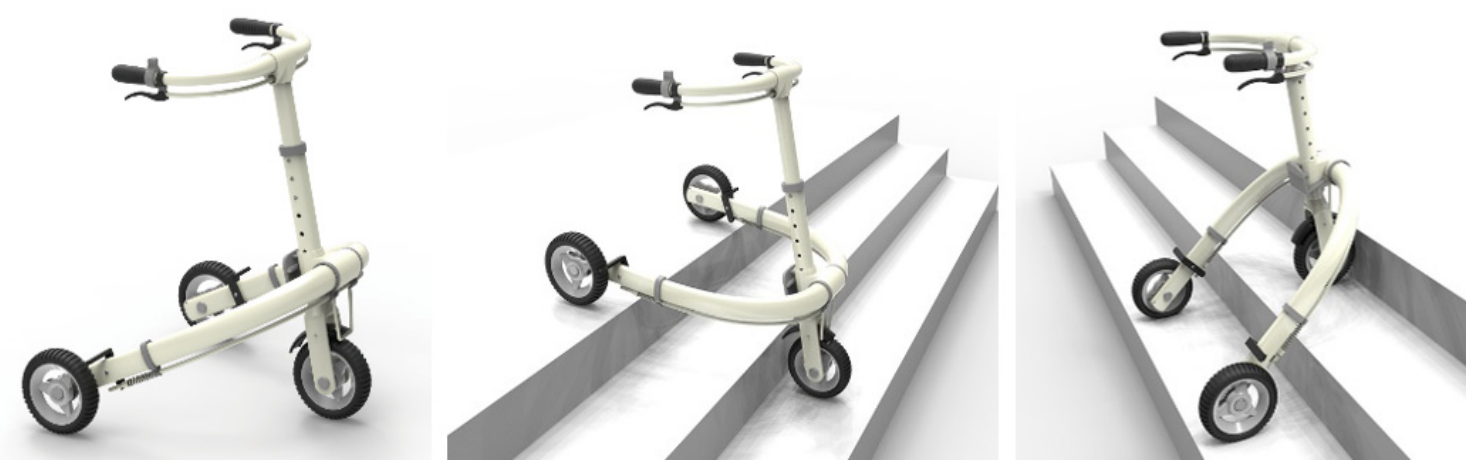

Figure 9. Final design (second paper prototype) (walking on level ground / walking down the stairs / walking up the stairs)

This auxiliary walker has the following functions.

- Wheels do not roll over a certain speed, so that it can be used safely on level ground and downhill.

- Wheels can roll in only one direction, so that it can be used safely on uphill road.

- When going up the stairs, the wheels cannot roll backward for safe movement.

- When going down the stairs, brakes are locked automatically for safe movement.

- Height is adjustable according to the user's physical characteristics.

- It is easy to fold and easy to store.

- It is easy to maintain because of the mechanical design.

Figure 10 shows how to operate the Tri-Wheel Stair Walker during concrete movement.

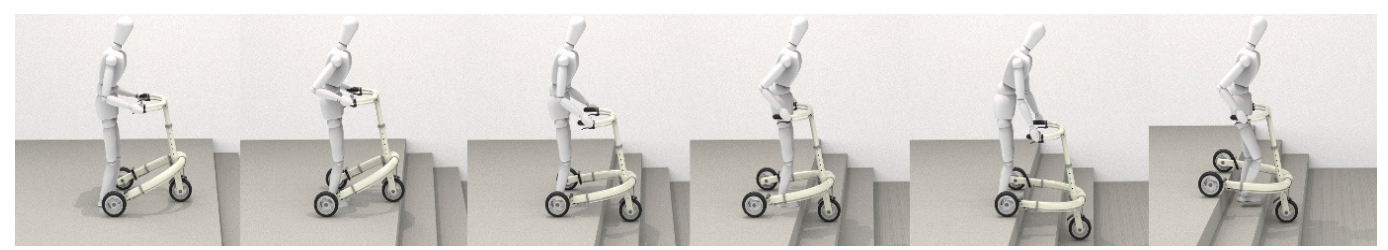

Walking on level ground $\rightarrow$ Walking down the stairs

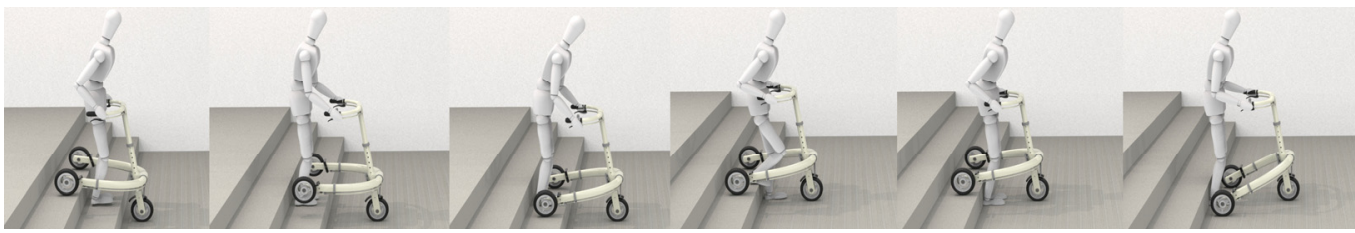

Walking down the stairs $\rightarrow$ Walking on level ground

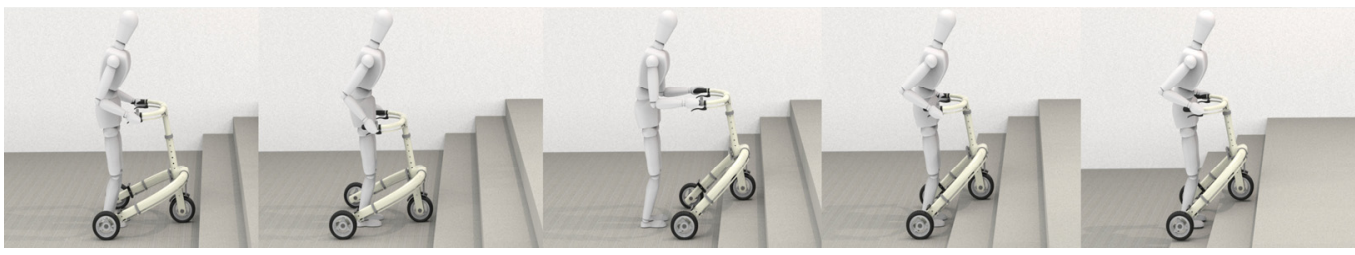

Walking on level ground $\rightarrow$ Walking up the stairs

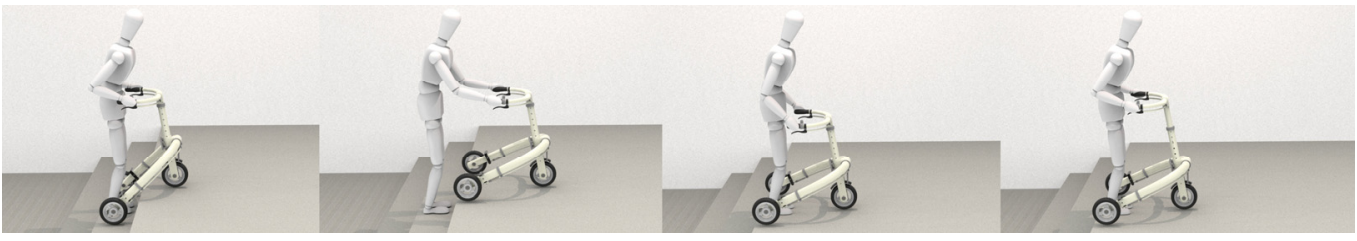

Walking up the stairs $\rightarrow$ Walking on level ground

Figure 10. Movement with Tri-Wheel Stair Walker 
Also, as shown in (Figure 11), it is easy to fold and easy to store, so that it can be used when going out with automobiles. Two or more walkers can be stacked and hence, they can be used in public facilities such as hospitals and nursing homes.

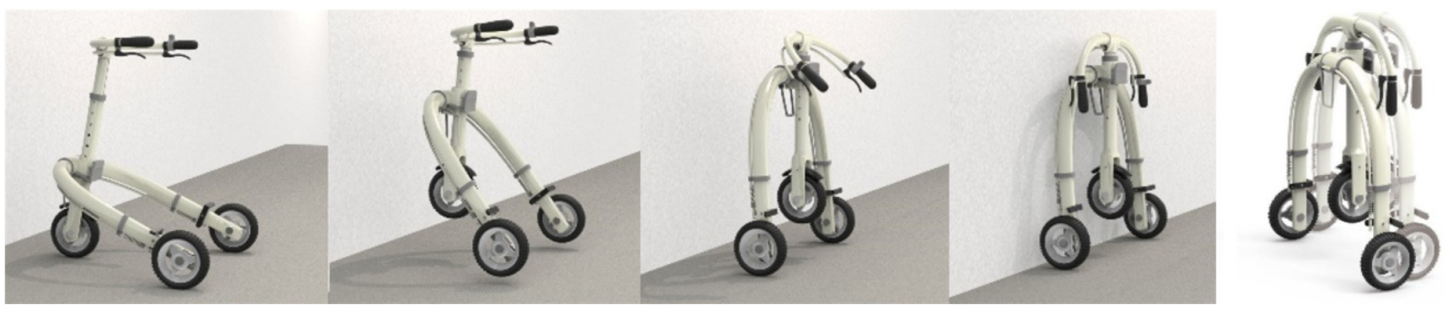

Figure 11. Folding

Regarding the details, the wheels do not roll over a certain speed, so that the walker can be moved safely on level ground or downhill. Because the wheels can only roll forward, the walker will not be pushed backward when going uphill. The wheels are designed as shown in Figure 12 (left). As shown in Figure 12 (middle), when going down the stairs, the angle of the frame changes from $90^{\circ}$ to $75^{\circ}$, and the brake is locked automatically. Also, as shown in Figure 12 (right), the height can be adjusted according to the user's physical characteristics. Above all, there is no electronic device and no need to use a battery. Additionally, the mechanical brake design, which can be automatically caught by the change of the frame angle, and the wheel design, which can roll at a constant speed only in one direction, make the walker easy to maintain.
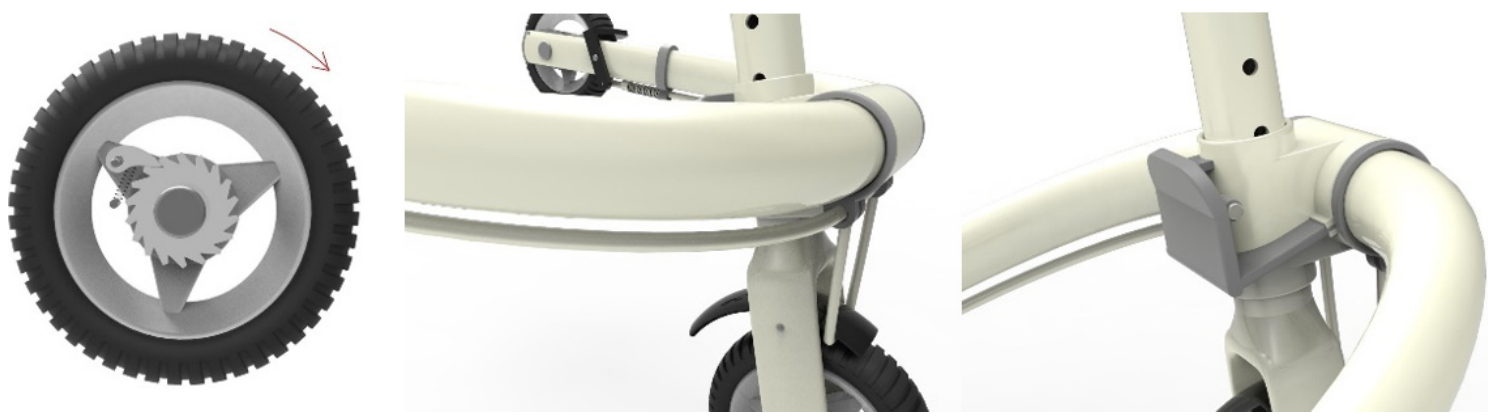

Figure 12. The wheel, which can only roll in one direction (left) / The mechanical brake, which can be locked automatically on downhill (middle) / The frame, which is flexible to adjust to height and angle (right)

\section{Discussion}

In this study, we investigated the characteristics of the movement of the elderly and analysed the walking assist products on the market, also proposing the design concept Tri-Wheel Stair Walker based on interviews with and observation of elderly users. We evaluated its practicability using pilot test and designed a structure that needs no battery and can be easily maintained by employing structural prototypes.

As shown in Figure 13, we utilized the principles of human-centered design and proposed a new auxiliary walker, which can reduce users' psychological burden and provide users with a desirable doorto-door mobility solution. We also connected mechanical principles with user behaviors, rendering possible the safe movement on stairs and slopes. The walker without electronic units can be manufactured at a low cost and can also easily be turned into products.

In this follow-up study, we will manufacture the walker and by conducting further user evaluation, we will verify the design and engineering practicability and will finally develop it into a real product to assist the elderly in their movement activities. The concept proposed in this study is a walker which can be purchased at an affordable price. It will help the elderly who have high mobility needs but walk with 
difficultly to improve their mobility, and help those who are reluctant to go out because of the stairs to improve their right to access. It is expected to be a safe, healthy and cheap solution to the door-to-door mobility needs in a smart city.

\section{Reduce users" psychological burden}

Provide the users a desirable door to door mobility solution

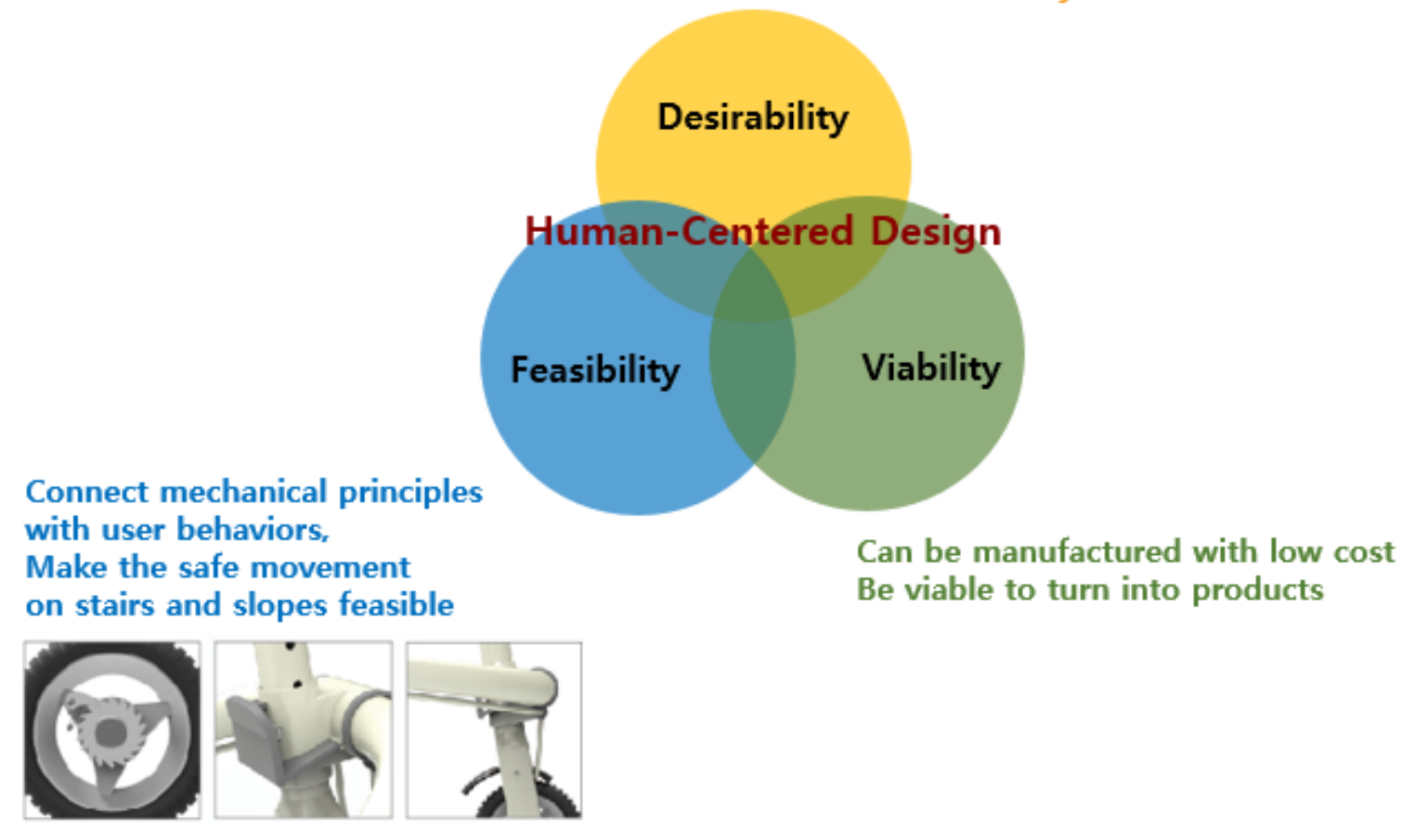

Figure 13. Characteristics of Tri-Wheel Stair Walker in the perspective of humancentered design

\section{References}

AGE Platform Europe (2002), Door-to- door transport systems: older people's point of view. [online] AGE - The European Older People's Platform / La plate-forme Eurpéenne des Personnes Agées. Available at: https://www.age-platform.eu/policy-work/news/age-door-door-transport-systems-older-peoples-point-view (accessed 11.10.2017).

Bonneville, J-B. (2016), "Deriving new business models for door-to-door mobility", Proceedings of the $14^{\text {th }}$ World Conference on Transportation Research (WCTR 2016), Shanghai, China, July 10-15, 2016.

Fricke, J. and Unsworth, C.A. (1997), "Inter-rater reliability of the original and modified Barthel Index, and a comparison with the Functional Independence Measure", Australian Occupational Therapy Journal, Vol. 44 No. 1, pp. 22-29. https://doi.org/10.1111/j.1440-1630.1997.tb00750.x

Jung, H.Y., Park, B.K., Shin, H.S., Kang, Y.K., Pyun, S.B. et al. (2007), "Development of the Korean version of Modified Barthel Index (K-MBI): multi-center study for subjects with stroke", Journal of Korean Academy of Rehabilitation Medicine, Vol. 31 No. 3, pp. 283-297.

Kim, A.J., Kweon, O.S. and Shin, C.B. (2017), "A Study on Classification of User Interface of Mobility Assistive Products for People with Disability", Journal of the Korean Society of Design Culture, Vol. 23 No. 1, pp. 111122.

Kim, G.-D., Kim, S.-S. and Han, G.-S. (2012), "Kinematic Analysis of Weight Distribution and Stair Descent Gait Motions Between Obese group and Non obese group of Elderly Women", Journal of Coaching Development, Vol. 14 No. 3, pp. 95-104.

Lee, J.S. (2008), A study on robot design for aiding elderly's outdoor movement: based on ADL (Activities of daily living) research, Master thesis, Hong-ik University. 
Roy, M.S. (2001), "Serious stair injuries can be prevented by improved stair design”, Applied Ergonomics, Vol. 32 No. 2, pp. 135-139. https://doi.org/10.1016/S0003-6870(00)00049-1

Meile Le, Master Student

Seoul National University, Graduate School of Design

1 Gwanak-ro, Gwanak-gu, 151742 Seoul, South Korea

Email: lemeile203@gmail.com 